\title{
Editorial
}

\section{Nanomaterials Processed by Spark Plasma Sintering}

\author{
Faming Zhang, ${ }^{1}$ Bikramjit Basu, ${ }^{2}$ Lianjun Wang, ${ }^{3}$ \\ Izabel Fernanda Machado, ${ }^{4}$ and Claude Estournès ${ }^{5}$ \\ ${ }^{1}$ Physics of New Materials, Institute of Physics, University of Rostock, 18055 Rostock, Germany \\ ${ }^{2}$ Materials Research Center, Indian Institute of Science (IISc), Bangalore 560012, India \\ ${ }^{3}$ College of Materials Science and Engineering, Donghua University, Songjiang District, Shanghai 201620, China \\ ${ }^{4}$ Departamento de Engenharia Mecatrônica e Sistemas Mecânicos, Escola Politécnica da Universidade de São Paulo, \\ 05508-900 São Paulo, SP, Brazil \\ ${ }^{5}$ Institut Carnot CIRIMAT (UMR 5085 CNRS-UPS-INP), Université Paul Sabatier, 118 Route de Narbonne, \\ 31062 Toulouse Cedex 09, France
}

Correspondence should be addressed to Faming Zhang; faming.zhang@uni-rostock.de

Received 17 November 2013; Accepted 17 November 2013

Copyright (C) 2013 Faming Zhang et al. This is an open access article distributed under the Creative Commons Attribution License, which permits unrestricted use, distribution, and reproduction in any medium, provided the original work is properly cited.

The spark plasma sintering (SPS), a variant of field-assisted sintering (FAST) or pulsed electric current sintering (PECS), is a novel pressure assisted pulsed electric current sintering process, which utilizes ON-OFF DC pulse energizing. Due to the repeated application of an ON-OFF DC pulse voltage and current flow between powder particles, the spark discharges and the Joule heating (local high temperature state) are therefore dispersed to the overall specimen. The SPS process is based on the electrical spark discharge phenomenon and is a high efficient, energy saving technique with a high heating rate and a short holding time. The problem of rapid grain growth of nanomaterials during conventional sintering can be inhibited to a larger extent by using the SPS technique. The SPS can be used for diverse novel bulk material applications, but it is particularly suitable for the processing of nanomaterials. Despite such anticipated advantages, the optimization of the process window (heating rate-temperature-time) in SPS process is a challenging task. More importantly, the underlying mechanisms for superfast densification still remains to be explored.

In the above perspective, this special issue contains some peer-reviewed research papers, which address some of the exciting issues or illustrate new nanomaterials development using SPS. This special issue's papers are all very much of the "head up" variety. First D. V. Dudina and A. K. Mukherjee reviewed the reactive SPS on the successes and challenges of nanomaterial synthesis. Secondly, some work on bioceramics is covered, for example, antimony-doped tin oxide nanoceramics (J. Wu et al.). Additionally, we look to some works on the nanostructured metals, for example, nanostructured nickel (F. Naimi et al.) and FeAl alloys (S. Paris et al.). In the end, the SPS of nanocomposites is also introduced, for example, graphene $/ \mathrm{Bi}_{2} \mathrm{Te}_{3}$ thermoelectric materials (B. Liang et al.) and $\mathrm{Ti}-\mathrm{ZrO}_{2}$ functionally graded materials ( $\mathrm{M}$. Jayachandran et al.).

This field of "nanomaterials processed by SPS" develops very fast. This special issue is just a tip of the iceberg, but we also can conjure up the whole thing through seeing a part of it.

We thank all the authors for their contribution to the literature on SPS.

Faming Zhang Bikramjit Basu Lianjun Wang Izabel Fernanda Machado Claude Estournès 

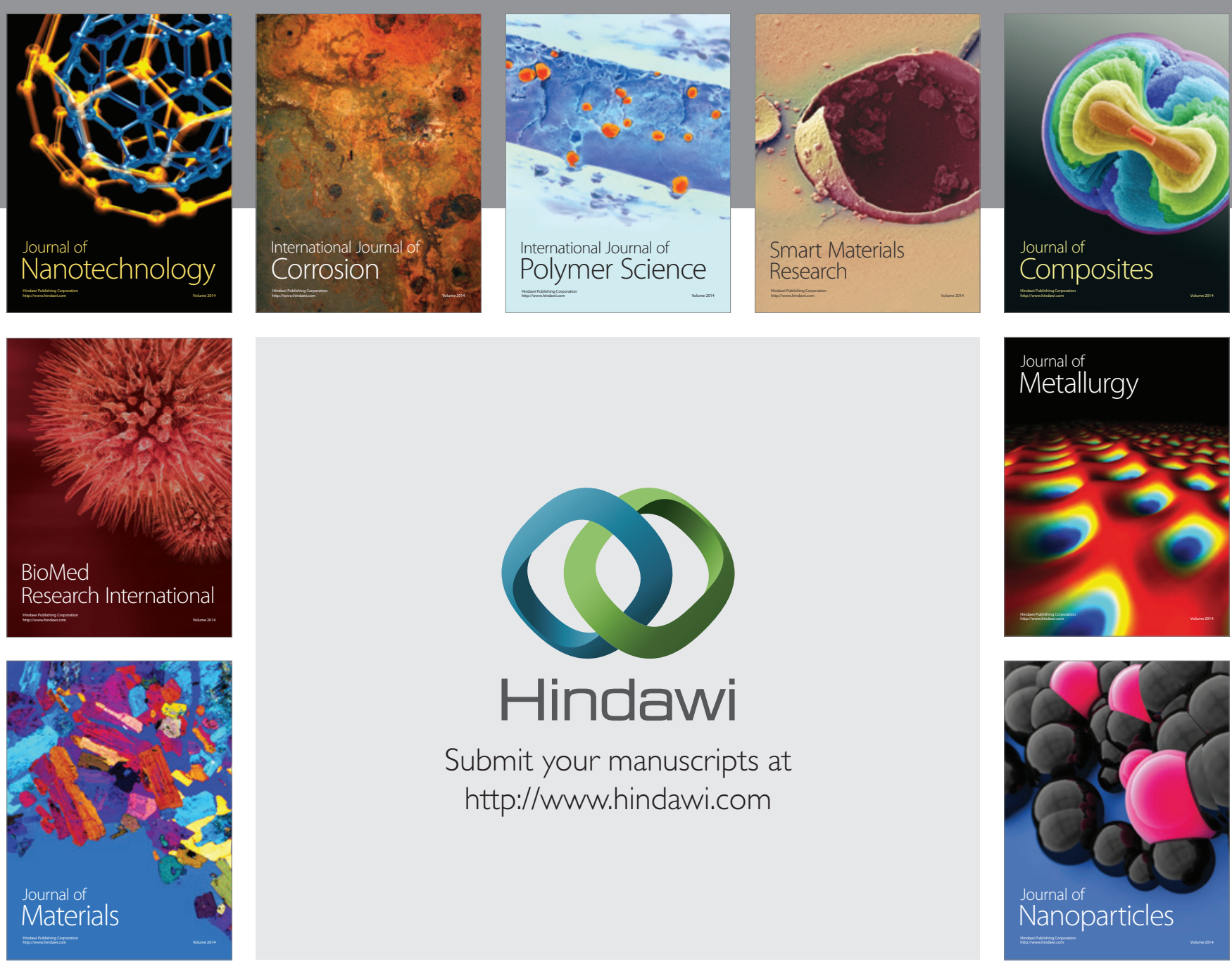

Submit your manuscripts at http://www.hindawi.com
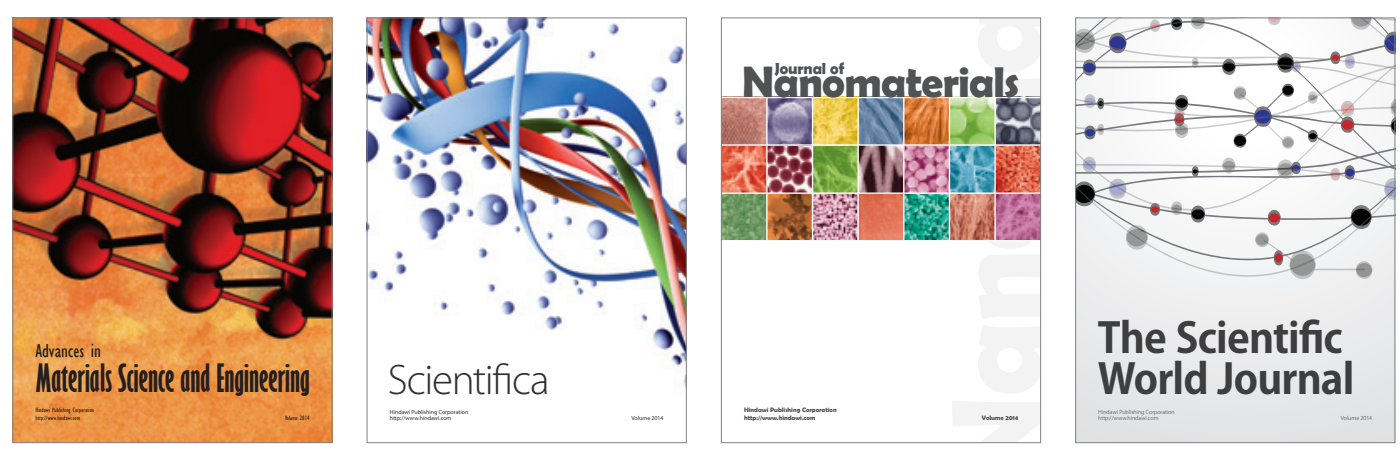

\section{The Scientific World Journal}
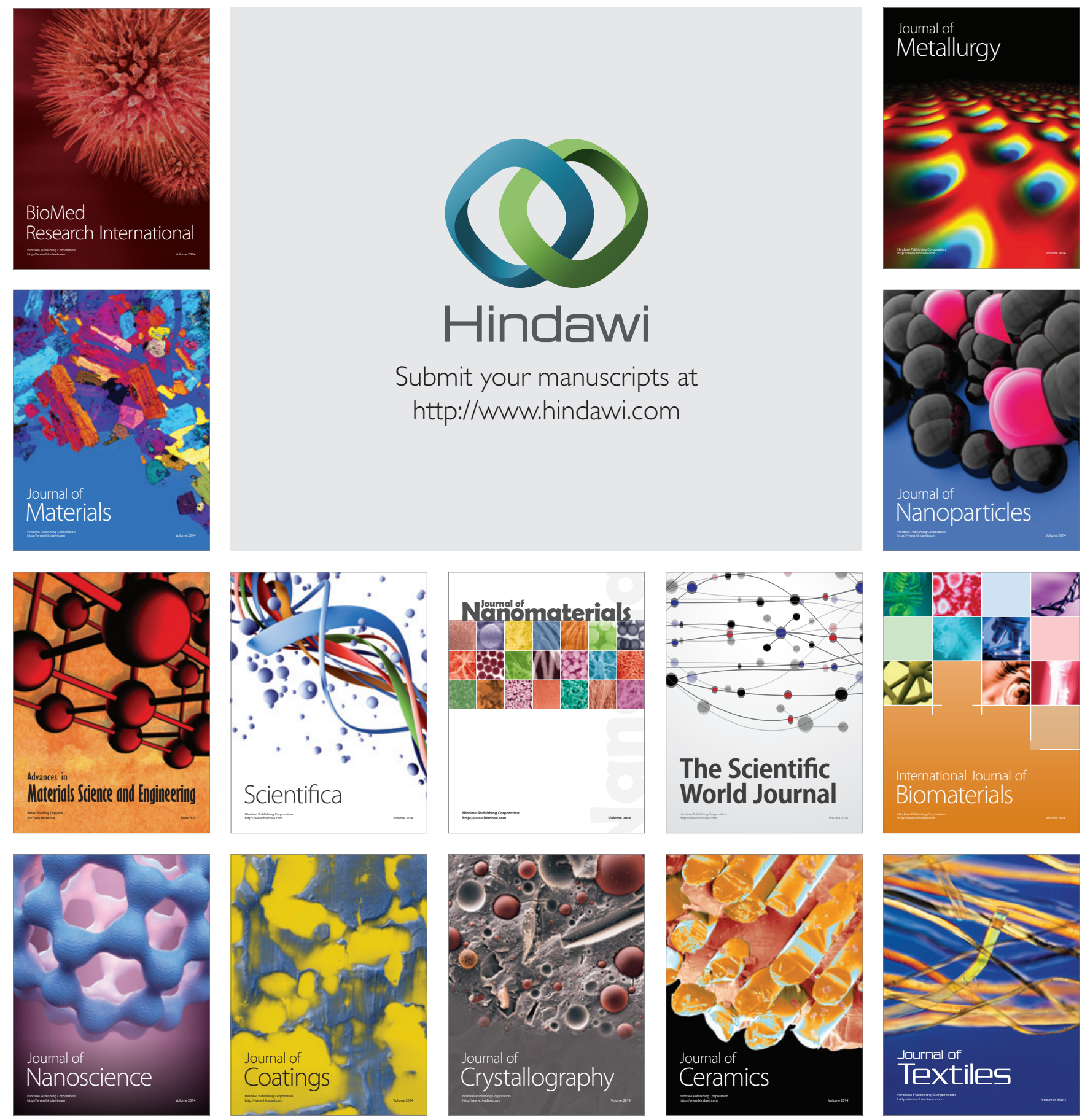